Voix et Images

\title{
La dimension pathémique du discours poétique
}

\section{Roger Chamberland}

Volume 16, numéro 3 (48), printemps 1991

François Charron

URI : https://id.erudit.org/iderudit/200921ar

DOI : https://doi.org/10.7202/200921ar

Aller au sommaire du numéro

\section{Éditeur(s)}

Université du Québec à Montréal

\section{ISSN}

0318-9201 (imprimé)

1705-933X (numérique)

Découvrir la revue

\section{Citer cet article}

Chamberland, R. (1991). La dimension pathémique du discours poétique. Voix et Images, 16(3), 456-468. https://doi.org/10.7202/200921ar d'utilisation que vous pouvez consulter en ligne.

https://apropos.erudit.org/fr/usagers/politique-dutilisation/ 


\section{La dimension pathémique du discours poétique}

\section{par Roger Chamberland, université Laval}

D'où vient l'émotion ? ${ }^{1}$ demande le poète François Charron dans le Monde comme obstacle. Question posée, question laissée en suspens avec comme élément de réponse possible cette petite phrase qui termine ce même recueil: On dit la vérité sans la trouver. ${ }^{2}$ Autrement posée, rapprochant cette fois-ci l'un et l'autre terme, la question deviendrait: l'émotion réside-t-elle dans la recherche de vérité? Reprise encore, retournant les termes, on dirait: la recherche de la vérité est-elle à la source de l'émotion? L'émotion, comme la vérité, peut-elle exister en dehors de la conscience qui en constate l'avènement et la manifestation? ou, comme l'écrit Charron dans La beauté des visages ne pèse pas sur la terre: il n'y a pas de vérité lointaine, tout est toujours là. ${ }^{3}$ On trouverait dans la poésie classique et moderne une réponse satisfaisante où, tour à tour, les poètes se sont employés à approfondir cet acte de conscience à partir d'un lyrisme assorti à un champ émotionnel dont plusieurs critiques et commentateurs ont fait l'inventaire. Mais "l'extrême contemporain", pour reprendre un nom de collection de poésie et d'essais publiée en France, cette poésie publiée depuis quelques années - ne pourrionsnous pas également étendre notre domaine d'étude à la musique par exemple ou au théâtre? - peut-il également être investi par l'émotion, lors même que la recherche formelle accapare souvent l'avantscène et devient le lieu de butée de la lecture et de la critique? En bref, d'où vient l'émotion si l'harmonie du son, des sens et du langage ne converge plus de façon évidente, comme c'était le cas traditionnellement, vers une plus grande unité, vers une représentation homogène et intégrée d'un état d'âme? Dans la fragmentation, le tumulte, le désordre, existe un chaos fondamental autour duquel s'organisent l'émotion fragile, la pensée créatrice, la réflexion intellectuelle. La théorie du "big bang " à l'origine de la création de l'univers

1 François Charron, le Monde comme obstacle, Montréal, les Herbes rouges, 1988, p. 184.

2 Ibid., p. 130.

3 François Charron, La beauté des visages ne pèse pas sur la terre, TroisRivières, Ecrits des forges, 1990, p. 78. 
n'est-elle pas, elle aussi, une façon de concevoir l'existence actuelle à partir d'un grand magma cosmique indifférencié?

[...] un homme se tient la tête, un homme observe la terre qui s'ouvre $^{4}$ : geste inaugural et apparition d'une dimension inédite, premiers instants de poésie de la Fragilité des choses, plaquette de 36 textes de François Charron. Qui est cet homme penché, cet observateur qui fixe le dévoilement d'un univers livré dans l'attraction de la terre, dans la brisure du livre? Ailleurs: Qui parle encore à travers les tourments? (p: 38) et la tombée finale: Lintériorité qui nous donne une enfance n'est pas parfaitement pure. Elle se brise, elle est déjà brisée. La substance au loin attend qu'une même histoire fasse partie de nous. (p. 50) Passage continuel d'un monde naturel à un monde abstrait et interrelation de l'un à l'autre. La poésie, en effet, loge-t-elle à ce croisement de deux mondes qui se désirent mutuellement et s'éprouvent dans la véridiction de leurs énoncés? Lidée d'un seul monde est une idée imparfaite, nous répète Charron dans le Monde comme obstacle $^{5}$. Mais encore faudrait-il être certain que le monde naturel existe en dehors de nous, hors de l'expérience qui nous en signale la présence par la sensation que nous en éprouvons. Celle-ci ne peut être qu'approximative, entachée d'antécédents de toute nature qui en modifient la structure première. Merleau-Ponty souligne à juste titre que:

La théorie de la sensation, qui compose tout savoir de qualités déterminées, nous construit des objets nettoyés de tout équivoque, purs, absolus, qui sont plutôt l'idéal de la connaissance que ses thèmes effectifs, elle ne s'adapte qu'à la superstructure tardive de la conscience. [...] Les images que l'instinct projette devant lui, celles que la tradition recrée dans chaque génération, ou simplement les rêves se présentent d'abord à droits égaux avec les perceptions proprement dites, et la perception véritable, actuelle et explicite, se distingue peu à peu des phantasmes par un travail critique. ${ }^{6}$

L'aspect véridictoire de la sensation n'est possible que dans l'exercice d'un travail critique issu de l'émotion. Toute recherche d'un surcroît d'origine ne peut provenir que d'une tension émotionnelle qui présuppose un repli de l'émotion sur elle-même, et donc un creux intérieur producteur. Encore faut-il que cette émotion soit convertie en passion, c'est-à-dire qu'il y ait affranchissement du psychologisme caractéristique de l'émotionnel qui ne laisse pas le sujet parvenir à la réflexion ${ }^{7}$. Kant, repris par Parret, définit mieux encore la passion comme étant

4 François Charron, la Fragilité des choses, Montréal, les Herbes rouges, 1987, p. 7.

5 Id., le Monde comme obstacle, op. cit., p. 24.

6 Maurice Merleau-Ponty, Phénoménologie de la perception, Paris, Gallimard, 1976, p. 78-79.

7 Emmanuel Kant, Critique de la faculté de juger, Paris, Vrin, 1984, p. 48. 
l'inclination que la raison du sujet ne peut maîtriser ou n'y parvient qu'à peine, l'inclination étant le désir sensible habituel, et le désir sensible habituel, et le désir d'autodétermination du pouvoir d'un sujet par la représentation d'un fait futur qui serait l'effet futur de ce pouvoir ${ }^{8}$.

Sensation, émotion, passion et désir sont les clefs de voûte de l'être-au-monde et peut-être plus encore de l'écrivain, du poète qui, plus directement, tente de mettre en discours sa subjectivité et de la communiquer en prenant appui sur des éléments qui pourraient être facilement partagés. Voilà qui relève du lieu commun bien que, dans l'histoire de la poésie, la subjectivité ait été suspecte aux yeux de certains comme, par exemple, durant la période "formaliste" des Herbes rouges à laquelle adhérait François Charron. Pour appréhender cette subjectivité en poésie, les études littéraires privilégient la thématique et/ou la phonétique s'employant à établir la typologie des indicateurs discursifs de la subjectivité plutôt que de montrer comment la subjectivité est mise en discours. Notre objectif ici est double: d'une part, levant l'objectivisme propre à la sémiotique des modalités, on développera le postulat heuristique suivant, à savoir que la poésie est l'exemplification de la mise en discours de la subjectivité puisqu'elle est fortement marquée par la figurativité et par la rhétorisation et qu'elle provient, $a$ fortiori, de l'identité réciproque entre un "je" énonciatif et un "je" anthropomorphe (auteur). D'autre part, il nous semble que la poésie de François Charron, depuis le milieu des années 1980, offre un lieu d'exercice pour une sémiotique des passions dans la mesure où les indicateurs discursifs de la passion en sont presque absents, laissant place à un discours où la subjectivité doit nécessairement loger à un niveau beaucoup plus profond. Nous retracerons le parcours de l'enthousiasme et de la reconnaissance, considérés comme des "passions de passions", en parlant dú recueil le Fait de vivre ou d'avoir vécu (1986) qui marque, tant d'un point de vue formel que thématique, une proposition poétique inédite, jusqu'à La beauté des visages ne pèse pas sur la terre (1990). Cinq recueils caractérisés principalement par un plus grand dépouillement de l'écriture, une présence "sublime" du quotidien et une attention particulière aux objets qui le composent. Comme l'écrivait Émile Zola dans le Roman expérimental: sans doute nous sommes loin ici des certitudes de la chimie. Nous ne connaissons point encore les réactifs qui décomposent les passions et qui permettent de les analyser. ${ }^{9}$

8 Emmanuel Kant cité dans Herman Parret, les Passions. Essais sur la mise en discours de la subjectivité, Bruxelles, Pierre mardaga éditeur, 1986, p. 42.

9 Émile Zola, le Roman expérimental, Paris, Garnier-Flammarion, 1971, p. 21. 


\section{Une logique du pathos}

Pour Greimas, les règles de l'organisation diégétique sont affectées par la catégorie thymique entendue comme:

une catégorie "primitive», dite aussi proprio-ceptive, à l'aide de laquelle on cherche à formuler, très sommairement, la manière dont tout être vivant, inscrit dans un milieu, "se sent" lui-même et réagit à son environnement, un être vivant étant considéré commé un "système d'attractions et de répulsions $» .10$

Le thymique, du grec thumos, le siège des passions, le cœur, l'affectivité, désigne cette catégorie, provenant de la théorie des modalités suggérée par Greimas et hiérarchisée au niveau narratif entre le pragmatique et le cognitif. Dès lors, plusieurs chercheurs lui ont donné une meilleure assise théorique ${ }^{11}$ à partir de laquelle on peut fonder une analyse rigoureuse de la dimension pathémique du discours poétique. Relevant l'ancienne opposition Logos-Pathos, on dira plutôt que les deux termes sont en relation binaire: la raison est toujours marquée par des pathèmes, ou éléments passionnels, le pathos subissant quant à lui tout autant lés contraintes d'une raison dont il ignore la logique, selon l'adage connu: le cœur a ses raisons que la raison ignore.

La sémiotique subjectale prend appui sur la pathémisation des discours ou, plus exactement, sur les mécanismes de la mise en discours des passions. Il faut alors considérer que la performativisation et la figurativisation des discours sont des générateurs extrêmement puissants du pathos en pleine émergence. Parret souligne à juste titre que:

Le sujet dans le discours, [...] n'est pas celui qui "dit vrai» ou "croit vrai", mais un être de passion. On a longtemps fait comme si celui qui parle ne déployait que des activités cognitives. C'est celui qui pense et énonce sa pensée, c'est celui qui nomme, décrit, affirme, constate, raisonne, conclut. ${ }^{12}$

L'exigence d'un objectivisme structural a longtemps empêché que les niveaux cognitif et pragmatique puissent avoir une sorte de zonetampon où les passions joueraient en quelque sorte le rôle de rotule. La logique des passions est à reconstituer à partir d'un effort d'interprétation portant sur l'instance d'énonciation qui doit être mise au jour afin de reconstituer la compétence passionnelle. Il serait contreindiqué de confondre la passion et les indicateurs discursifs de cette passion. L'effort d'interprétation devra porter plus spécifiquement

10 A..J. Greimas, Du sens II, Paris, Seuil, 1983, p. 93.

11 Notamment celle de Herman Parret, le Sublime du quotidien, Paris et Amsterdam, Hadès-Benjamin, 1988.

12 Herman Parret, les Passions, op. cit., p. 150. 
sur la performativisation et la figurativisation comme nous l'avons déjà signalé. On entend par performativisation la manière avec laquelle une force émotive laissera son empreinte sur le texte, l'énoncé, le terme ou le syntagme. Cette force émotive (ou tension de production) s'ajoute paratactiquement à la signification d'une expression; cette signification est complète et achevée lorsque cette expression a un sens et une référence. Cette force, qui peut être de plusieurs natures, est reliée plus spécifiquement à la signification émotive en soi, un type de signification qui échappe au critère de vérification à partir de n'importe quelle évidence empirique. La fonction qui s'y rattache est l'expression et l'évocation de sentiments et d'attitudes; elle est donc tout à fait contraire à la fonction symbolique ou référentielle qui acquièrent toujours une valeur de vérité. Dans ce contexte, l'émotion apparaît comme un opérateur de force qui modifie tous les contenus, même ceux qui sont contenus dans des assertions, des phrases déclaratives ou des figures de style. Cette proposition nous permet d'accorder à la rhétorique un rôle de premier plan dans la performativisation puisqu'elle circonscrit la manière d'être de l'énonciation inventée - disposition - élocution.

Quant à la figurativisation, elle sous-tend, à l'instar de la force émotive, une force figurative qui agit de la même manière sur tout texte, tout énoncé et tout syntagme. Dès qu'il y a investissement sémantique dans un programme narratif, il y a figurativisation, naissance d'un monde à contours figuratifs, soit par l'actorialisation, la spatialisation ou la temporalisation. Je mets de côté toute la discussion pour savoir à quel monde renvoie la poésie; car cela nous amènerait dans un débat philosophique visant à déterminer si la dimension pathémique peut exister dans un monde non figuratif.

\section{Présence du sublime}

On peut interroger quelques œuvres nouvelles, chercher en elles le lieu d'expression d'une force émotionnelle sous-jacente à toute pratique d'écriture et, plus largement, de toute manifestation artistique. Cette force participe à la caractérisation de la mise en discours de la dimension pathémique que la sémiotique est parvenue à mieux articuler après en avoir supputé l'existence durant de nombreuses années. Cette mise en discours de la subjectivité, principalement développée par Herman Parret, donne aux passions une assise théorique selon la théorie des modalités qui est au cœur de l'analyse sémiotique. Toutefois, le discours des passions ne saurait retenir notre attention si l'on ne prend pas en considération ce par quoi il advient: le sublime. Voilà ainsi constituée une chaîne signifiante qui va de l'émotion à la passion en passant par le sentiment du sublime.

Pour François Charron, comme pour de nombreux poètes québécois contemporains, le sentiment du sublime émerge du quotidien; 
il n'existe pas en dehors de ce qui constitue l'instant présent, le hic et nunc par lequel l'itérabilité perd sa consistance au profit d'une phénoménologie sans cesse renouvelée, assujettie à des conditions d'existence elles-mêmes tributaires d'une transformation incessante du monde ambiant. Comme le souligne à juste titre Herman Parret:

la quotidienneté n'est pertinente dans la vie que parce qu'elle enchâsse le sublime. La fracture du quotidien, la rupture de l'isotopie de la quotidienneté par les irruptions du sublime, c'est ce qui constituera le quotidien et le sublime comme pôles d'une délimitation réciproque. ${ }^{13}$

Le quotidien devient le lieu privilégié d'un questionnement sur le sens de la vie puisqu'il est continuellement dénié par l'émotion esthétique qui fait saillie là où l'on s'y attend le moins. À ce titre, le sublime est à départager du beau qui conforte en nous un sentiment d'intensification de la vie homogène et de non-contradiction tandis que le sublime est grandiose, colossal. [...] il force à l'admiration et au respect ${ }^{14}$, comme le rappelle Kant: Autrement dit, le sublime n'est possible que s'il est sous-tendu par la passion du sublime, le quotidien ne peut s'éprouver comme tel sans irruption et frémissement du sublime.

Depuis le début des années quatre-vingt, l'œuvre de François Charron s'est placée sous le signe de cette phénoménologie du quotidien et du sublime, dans la mesure où le sublime resémantise la vie quotidienne, les objets environnants usagés, les relations intersubjectives usées, selon les expressions heureuses de Greimas. Les titres de ses recueils sont à eux seuls fort significatifs: le Fait de vivre ou d'avoir vécu (1986), la Fragilité des choses (1987), le Monde comme obstacle (1988), La beauté pourrit sans douleur (1989) et La beauté des visages ne pèse pas sur la terre (1990). Dans chacun de ces livres, il procède à un inventaire tout à la fois partiel et significatif du monde qui l'entoure, lequel inventaire est largement tributaire du sublime plutôt qu'il ne se situe dans les marges de ce qui compose la réalité. Mais, plus que les titres, ce sont les textes eux-mêmes qui sont comme autant de noyaux de réalité, une réalité souvent banale, soumis à la contemplation et à la méditation:

cette valise est bien fermée

je suis assis quelque part

un nuage ne va plus redescendre ${ }^{15}$

Le poète extráit de son quotidien des éléments composites à partir desquels il ne tente pas de donner un sens à l'indéchiffrable

13 Herman Parret, le Sublime du quotidien, op. cit., p. 20.

14 Emmanuel Kant, op. cit., p. 68.

15 François Charron, La beauté pourrit sans douleur suivi de la Très Précieuse Qualité du vide, Montréal, les Herbes rouges, 1989, p. 143 . 
permanence $d u$ réel 16 tel qu'il ne présente à lui, mais essaie plutôt de donner le plus objectivement possible les trois facteurs ayant concouru à l'irruption et au frémissement du sublime, soit l'éventualité d'un départ ou une arrivée récente, l'attente et/ou l'inactivité, l'improbabilité d'une légère ondée et/ou la garantie d'une température plus clémente. Autrement dit, l'événement de l'être-au-monde devient l'avènement d'un état qui en transcende l'immédiateté. Il importe peu que l'on sache précisément s'il s'agit de l'une ou l'autre option, que notre acte de lecture reconstitue le paysage tel qu'il a été perçu par le poète. Kant, mieux que quiconque, a défini ce qu'il appelle l'âme d'une œuvre ou le principe vivifiant en l'esprit:

ces représentations de l'imagination qui donnent beaucoup à penser [...] sans qu'aucune pensée déterminée, c'est-à-dire de concept, puisse [leur] être adéquate, et que par conséquent aucune langue ne peut complètement exprimer et rendre intelligible. ${ }^{17}$

Ces pures intuitions sans concepts, ajoute Kant, tendent à quelque chose qui se trouve au-delà des limites de l'expérience, soit la métaphysique. De toute époque, l'enjeu du sublime aura été la présentation du métaphysique et c'est en la poésie, conclut Kant, que la fa. culté.des Idées esthétiques peut donner toute sa mesure ${ }^{18}$. La poésie serait, selon lui, l'art du sublime par excellence, mais cela veut-il dire pour autant que le sublime est antérieur à l'extase qui le fait advenir? Jean-Luc Nancy a très bien dégagé la logique du sublime qui est mise en opération dans l'œuvre d'art:

Le sublime est un sentiment, et plus qu'un sentiment au sens banal, il est l'émotion du sujet à la limite. Le sujet du sublime, s'il y en a un, est un sujet ému. C'est de l'émotion du sujet qu'il est question dans la pensée du sublime, de cette émotion que ni la philosophie du sujet et du beau, ni l'esthétique de la fiction et du désir ne peuvent penser: car elles pensent nécessairement et seulement dans l'horizon de la jouissance du sujet (et du sujet comme jouissance). Et la jouissance, en tant que la satisfaction d'une présentation appropriée, coupe court à l'émotion. 19

Le sublime saisit le poète, c'est-à-dire qu'il a lieu au moment précis où l'imagination est incapable de présenter un objet, un état existant, ne serait-ce qu'en principe, qui s'accorderait avec un concept, entendu littéralement par exemple dans cet extrait:

16 François Charron, le Fait de vivre ou d'avoir vécu, Montréal, les Herbes rouges, 1986, p. 65.

17 Emmanuel Kant, op. cit., p. 110.

18 Ibid., p. 126.

19 Jean-Luc Nancy, «L'offrande sublime », Du sublime, Paris, Bélin, 1988, p. 63. 
Nos expériences nous désertent.

Le monde est là tout de suite.

Chacun le ressent à sa façon.

(le Fait de vivre ou d'avoir vécu, p. 160)

On peut définir une esthétique du sublime comme étant ce qui désigne l'imprésentable dans la présentation elle-même ${ }^{20}$, pour reprendre les termes de Jean-François Lyotard caractérisant le postmoderne. Reste la forme reconnaissable de la poésie, bien que celle-ci soit également soumise à une hybridation des genres qui en garantit la légitimité et permet l'acquiescement à une pratique canonique à contenu zéro. Comprenons bien qu'il existe tout de même une démarche poétique, ici suggérée dans l'œuvre de Charron, à partir de laquelle on doit construire une signifiance qui découle du contexte issu de la lecture des textes.

Le poème précité, comme la majorité de ceux qui composent son œuvre récente, ne devient "lisible» qu'au prix d'un exercice de lecture qui serait à envisager à la fois sur un axe syntagmatique et paradigmatique. Nous devons, dans un premier geste heuristique, recomposer une "structure d'horizon"; puis, dans un deuxième mouvement, herméneutique celui-là, délimiter des aires de signifiance qui constitueront les limites de notre interprétation et leur fondement. Chaque opération lectorielle informe la précédente, la modifie et la transforme en profondeur: le sens ne tombe pas sous le coup de l'évidence. La lecture devient un processus de reconstruction à partir de multiples isotopies disséminées dans l'œuvre jusqu'à l'ordonnancement d'une structure de signification.

Les cinq recueils que nous avons privilégiés dans cette étude présentent des similitudes tant formelles que thématiques qui nous permettent de les considérer comme un bloc homogène ayant comme principe unificateur un vouloir (une intention) de savoir: la vie, le monde, la beauté, etc., comme nous le soulignent les titres. Ce vouloirsavoir (le désir) d'un "sujet instaurateur" porte sur un objet séducteur: le réel. Dans la majorité de ces poèmes, on note le passage intermittent d'un univers quotidien, souvent familier et urbain, à un monde plus abstrait, métaphysique, qui se dépouille petit à petit des éléments qui composent son milieu environnant.

Le "je " dominant dans presque tous les poèmes est en position d'attente: il observe, il regarde, il décrit, il analyse. S'il agit, c'est pour se remettre en question; les objets, les gens le forcent continuellement à s'interroger sur lui-même et sur ce qui l'entoure: Le matin, je suis tourmenté et je me demande: Où vont ces passants? Que

20 Jean-François Lyotard, le Postmoderne expliqué aux enfants, Paris, Éditions Galilée, 1986, p. 32. 
font-ils? [...] Les passants réussissent à ne plus me parler (la Fragilité des choses, p. 9). Les objets sont présentés de façon brute, sans aucun qualificatif qui en spécifie l'une ou l'autre dimension; ils composent cet univers figuratif que traverse cette grammaire du désir. L'autre y apparaît sous forme de silhouette, de personnage, d'ombre, d'individu, de sentinelle, d'ami; même la femme aimée, l'amante échappe à la description jouissive. Bref, l'identité même du "je» anthropomorphe et du "je» énonciatif est soumise à de perpétuelles remises en question, comme le suggère par ailleurs une citation de John Keats placée en exergue dans le Monde comme obstacle: Un poète n'a pas d'identité; il habite sans cesse un autre corps. (p. 139) Toute certitude étant battue en brèche; toute existence est définie en fonction même de la connaissance fragmentaire que l'on développe à partir de l'expérience que l'on en fait.

L'inventaire des objets et des personnes est hétéroclite: les murs, le miroir, le drap, la fenêtre, un jouet, l'herbe, une femme assoupie, l'ami croisé par hasard et que l'on n'avait pas vu depuis longtemps, le rêveur. Rien ne se passe vraiment; dans chaque poème, le "je" raconte de multiples expériences où tout dérive d'un monde naturel avant d'être reconstitué par l'épreuve du corps et l'expérience de l'écriture. Beaucoup de poèmes proposent cette structure de signification où les faits réels se transforment en "effets de réel»:

\section{je longe tranquillement une falaise}

je jette mon sac en bas

toutes nos réponses finissent aveugles

(La beauté pourrit sans douleur, p. 127)

Le corps est engagé dans un "conflit " avec le monde, relation entièrement médiatisée par l'écriture. En termes philosophiques, on dirait: le paraître du monde s'oppose à l'être du corps. Merleau-Ponty, dans sa Phénoménologie de la perception, a largement exploré cette dimension cognitive de l'expérience humaine. Mais ici ce n'est pas le discours du philosophe qui retient notre attention, c'est celui du poète Charron qui étend son champ d'action au quotidien parce que sa parole est sa pensée, comme le démontre fort justement MerleauPonty. La pensée est tournée vers l'appréhension d'un savoir et assure, dans le flux ininterrompu de son déroulement, la non-fixité de ses règles ou la permanence de son inachèvement. Autant le réel est sondé, cité à comparaître, autant celui-ci questionne en provoquant le "je " énonciatif dans l'ensemble de ses émotions brutes par quoi il advient dans sa présence au monde.

Lá coïncidence du "je" énonciatif et du "je» anthropomorphe dans les discours poétiques des présents recueils est alimentée par une double force émotive et figurative. La force figurative, soustendue par la figurativisation qui en est l'état achevé, anime la 
circulation incessante d'un monde à contours figuratifs ${ }^{21}$ (naturel) à un monde non figuratif ou à isotopie figurative.

D'autre part, la force émotive découlant de l'émotion travaille le texte de fond en comble, alors que la fonction qui en émerge est directement manifestée dans la nomination de sentiments et d'attitudes. Dans ces poèmes, qui seraient toutefois à considérer comme des "épisodes narratifs", nous avons un sujet de désir/d'obligation (existentiel) disjoint de l'objet, que nous pouvons investir de la valeur "vouloir-savoir" relevant de la modalisation virtualisante; dès lors, le discours sera défini par le programme narratif qui consiste à conjoindre le sujet à l'objet. La valeur précitée est convertie sémantiquement par la figure de la réalité quotidienne, d'où la figurativisation du discours poétique. En d'autres termes, on dirait que le sujet de désir/d'obligation vise une volonté de savoir contenue dans la réalité quotidienne, dans la mesure où son existence, le sens de sa vie et, par effet de retournement, de ce qui l'entoure sont entièrement tributaires de sa perception du monde. La quête de l'objet s'exerce précisément dans la pratique de l'écriture où l'énonciataire tente de circonscrire la réalité quotidienne afin de la confronter à l'exercice de ses sensations et perceptions. Toutefois, l'extraction du savoir de la réalité quotidienne ne peut être possible que dans la sublimation de celle-ci ou, comme le rapporte Parret:

Personne mieux que Greimas, dans De l'imperfection, n'a parlé de cette fracture que constitue l'apparition frémissante du sublime. "Bref instant d'indicible allégresse», immobilisation de l'objet-monde, éblouissement et guizzo-tressaillement, passage de la vue "ordinaire" à la vision "extra-ordinaire", instant de bonheur par la fascination de l'objet, surgissement du sublime. 22

Charron lui-même en fait la proposition formelle dans un de ses poèmes:

le ciel... la lumière... le vent

nous expérimentons la très précieuse qualité du vide

(La beauté pourrit sans douleur, p. 168)

Entendons ici que le savoir est constitué par cette métaphysique dont nous parlions au début, cet au-delà des limites de l'expérience qui aboutit au sentiment de plénitude propre aux contemplatifs et, plus généralement, à la philosophie orientale.

21 Nous sommes toutefois conscient de l'équivoque de ce terme qui impliquerait que le monde non figuratif est équivalent à abstrait. Le discours poétique ne pourrait souffrir longtemps de cette ambiguité.

Herman Parret, le Sublime du quotidien, op. cit., p. 20. 


\section{L'enthousiasme comme passion de la connaissance}

Il y a donc une fonction du sublime qui se rapporte à un principe subjectif, d'une part en relation avec la sensibilité, dans la mesure où elle favorise l'entendement contemplatif, d'autre part en opposition avec la sensibilité, de par son rapport aux fins de la raison pratique.

Ainsi, le sublime doit toujours avoir un rapport à la manière de penser, c'est-à-dire à des maximes qui visent à procurer à ce qui est intellectuel et aux idées de la raison la domination sur la sensibilité. 23

Le sublime peut se définir par la maîtrise d'une sensibilité qui manifeste l'espoir et l'inquiétude, deux passions chiasmiques issues d'une modalisation combinant le vouloir et le savoir; l'espoir associant vouloir-savoir et l'inquiétude vouloir-non-savoir selon la typologie de Parret ${ }^{24}$. Toutes deux peuvent être dites véridictives, car elles portent sur le contrat de vérité de l'objet de valeur. C'est-à-dire que le désir est un vouloir fondateur, instaurateur, qui subsume le vouloirsavoir dans la mesure où ce dernier est en voie d'actualisation, donc de transformation du discours. Dans cette quête de l'identité du monde environnant devenue aléatoire, la modalisation du vouloirsavoir présente dans l'espoir et l'inquiétude sous-tend une métamodalisation, c'est-à-dire que le vouloir se situe au deuxième degré. Cette métamodalisation qui conjugue une volonté désirante de vouloir-savoir a largement été décrite par Kant, et plus récemment par Parret: c'est l'enthousiasme, défini comme une passion de la passion théorique par excellence ${ }^{25}$; théorique parce que le vouloirdésir a pour objet le vouloir-savoir en tant que passion. Autrement dit, la réalisation est continuellement réinterprétée sous l'impulsion d'une force émotive qui traverse l'épaisseur du texte pour atteindre le discours.

L'enthousiasme est l'approfondissement de la passion qui reconduit et recherche ad infinitum l'objet de savoir, soit le vouloir-savoir, sans que rien, ni temps ni espace, ne vienne interrompre le déploiement de la pensée. L'écriture est à la fois le lieu d'écoulement de cette pensée effervescente et le lieu de son accomplissement. Au demeurant, l'enthousiasme a toujours été considéré comme l'exaltation du poète sous l'effet de l'inspiration comme nous le confirme le Petit

23 Emmanuel Kant, op. cit., p. 224.

24 Herman Parret, les Passions, op. cit. Dans cet essai fort important, Parret identifie trente-six passions principales réparties en trois grands types: chiasmiques (combinant vouloir et savoir), orgasmiques (relevant du devoir et du pouvoir) et enthousiasmiques, celles-ci étant des métamodalisations puisqu'elles sont de deuxième degré, car elles caractérisent la compétence passionnelle au niveau des préconditions de possibilité de l'univers pathique. Ibid., p. 189. 
Robert: C'est également, si l'on renvoie à l'étymologie du mot, l'enthousiaste qui apparaît vidé de lui-même; c'est l'Autre qui est Je (cf. les Mystiques et les Romantiques comme Schelling et Nietzsche). Ce vide subjectif implique une relation au sublime, au fait que l'imagination se sente illimitée en se représentant l'infini.

\section{L'écriture: passion de la reconnaissance}

L'enthousiasme, comme passion théorique, s'installe dans un lieu d'achèvement où la quête de l'objet - le vouloir comme intention de savoir - est continuellement subordonnée au vouloir-désir, dans la mesure où un désir qui atteindrait la fin de sa quête, donc qui assimilerait l'objet, s'abolirait dans la vacuité de son expérience puisque le vouloir-désir est la prime valeur.

Le parcours passionnel de l'enthousiasme, propre à la dimension cognitive, s'inscrit dans l'écriture poétique, qui en réalise la dimension pragmatique, afin de ne pas épuiser le sens de la quête de l'objet. En d'autres termes, on dira que l'écriture poétique place le sujet dans l'obligation de devoir-pouvoir (écrire) afin de reconnaître le topoï de son vouloir-désir (le monde).

La reconnaissance est cette passion de contrepartie qui, au niveau pragmatique, accompagne l'enthousiasme dont la fonction, selon Parret, est une fonction esthétique. C'est la saisie d'un objet par l'esprit, par la pensée, qui relie entre elles des images, des perceptions qui les concernent: distinguer, indentifier, connaitre par la mémoire, le jugement ou l'action, etc. Elle peut être définie comme le devoir-obligation dont l'objet est le devoir-nécessité de pouvoir, tandis que l'enthousiasme est le vouloir-désir dont l'objet est le vouloirintention de savoir. On en déduira que le désir de savoir, de connaissance du monde, est en conjonction avec l'obligation de pouvoir écrire. Le sujet instaurateur est un sujet de désir et d'obligation que l'on ne peut penser que dans son universalité et sa non-empiricité. La reconnaissance et l'enthousiasme, deux passions essentielles l'une à l'autre, sont des modalisations structurantes.

\section{Le bonheur: un appel au sens commun}

De fait, nous devrions considérer, comme nous le suggère Parret, que l'enthousiasme et la reconnaissance sont les passions les plus "élevées", puisque l'objet de ces passions est la passion même ${ }^{26}$. Le désir de l'intention de savoir et l'obligation de la nécessité de pouvoir sont les pré-conditions de l'émergence de l'écriture poétique. On peut ainsi établir que, dans un mouvement ultime, la connaissance du

26 Herman Parret, les Passions, op. cit., p. 80. 
monde induite par l'expérience de l'écriture est continuellement abolie ou plutôt régénérée par de nouveaux parcours figuratifs, ce que Charron décrit en ces termes: le poème qui m'accompagne est sa propre défaite (La beauté des visages ne pèse pas sur la terre, p. 21). L'écriture poétique met en œuvre une compétence passionnelle qui prend appui sur la faculté d'empathie, c'est-à-dire qu'il doit exister une communicabilité de l'univers des passions qui relève de l'ordre de la qualité de ce qui est communicable plutôt que du transfert d'information.

Dans les œuvres retenues, il y a un appel à un sens commun qui renvoie à l'esthétique plutôt qu'à la logique. Ne présentant aucune forme d'hermétịsme ou de rhétorisation excessive, ces textes se rapprochent du discours quasi descriptif, à la rigueur narratif. Ils participent d'un univers subjectif que l'on peut néanmoins facilement partager et qui sollicite notre adhésion quant à la reconnaissance du jugement esthétique qui en est fait. Cette sollicitation est à porter au compte de l'émotion et de la beauté, car elle manifeste le sens régulatif d'être-avec-les-autres ${ }^{27}$. C'est en ce sens que l'émotion et la beauté sont parties prenantes d'une passion communautaire, puisqu'elles peuvent être universellement communicables, donc qu'elles sont sens commun. Dès lors, reconnaître autrui, c'est estimer appartenir à la communauté, participer au sens commun. La reconnaissance [...] est un impératif catégorique dont la portée couvre le bonheur ${ }^{28}$. Sans contenu ni substance, le bonheur est une loi formelle qui définit notre acceptation d'être-avec-les-autres et s'immisce dans la vie passionnelle en dépit des contraintes esthétiques existantes. Charron écrit à ce sujet: le bonheur qui nous sauve se cache à la surface de nous-mêmes (La beauté des visages ne pèse pas sur la terre, p. 111). C'est dans la passion, là et seulement là, que le bonheur peut exister.

27 Ibid., p. 193.

28. Ibid. 